Data Article

\title{
Data set on effect of amaranth proteins on the RAS system. In vitro, in vivo and ex vivo assays
}

\author{
Santiago Suárez a, Paula Aphalo a, Gustavo Rinaldi ${ }^{\mathrm{b}}$, \\ Alejandra Quiroga a, *, María Cristina Añón a, ** \\ a Centro de Investigación y Desarrollo en Criotecnología de Alimentos (CIDCA-CONICET-CIC-UNLP), \\ Universidad Nacional de La Plata, La Plata, 1900, Argentina \\ ${ }^{\mathrm{b}}$ Facultad de Ciencias Médicas, Universidad Nacional de La Plata, La Plata, 1900, Argentina
}

A R T I C L E I N F O

\section{Article history:}

Received 2 December 2019

Received in revised form 4 January 2020

Accepted 15 January 2020

Available online 23 January 2020

\section{Keywords:}

Amaranth proteins

Protein hydrolysates

Bioactive peptides

Renin-angiotensin system

Antihypertensive emulsions

Spontaneously hypertensive rats

\begin{abstract}
A B S T R A C T
Data set presented in this article is related to the research paper entitled "Effect of amaranth proteins on the RAS system. In vitro, in vivo and ex vivo assays", available in Food Chemistry [1]. In this article, we evaluated the effect on systolic blood pressure of spontaneously hypertensive rats (SHR) of different samples with amaranth proteins/peptides. The effect of these samples on RAS system was evaluated using in vitro and ex vivo assays. The concentration of renin and angiotensin converting enzyme (ACE) was evaluated using two commercial ELISA kits. Renin concentration was estimated through a direct immunoassay and ACE concentration with an immunoassay based on a competitive inhibition. In addition, the ACE inhibitory activity in plasma was evaluated using a spectrophotometric assay according to [2]. Ex vivo experiments were done with thoracic aorta extracted during the surgical procedure employed to obtain blood samples according to [3]. Data presented in this article recollect a very extensive work on how can be affect the RAS system in SHR model using amaranth protein/ peptides as potential antihypertensive samples. These data could
\end{abstract}

DOI of original article: https://doi.org/10.1016/j.foodchem.2019.125601.

* Corresponding author.

** Corresponding author.

E-mail addresses: alejaquiroga@gmail.com, aquiroga@quimica.unlp.edu.ar (A. Quiroga), mcacidca@gmail.com, mca@biol. unlp.edu.ar (M.C. Añón). 
be useful to design novel functional foods for hypertensive individuals.

(1) 2020 The Author(s). Published by Elsevier Inc. This is an open access article under the CC BY license (http://creativecommons. org/licenses/by/4.0/).

Specifications Table

\begin{tabular}{|c|c|}
\hline Subject & Food Science \\
\hline \multirow[t]{2}{*}{ Specific subject area } & Bioactive peptides \\
\hline & Antihypertensive peptides \\
\hline Type of data & Table \\
\hline How data were acquired & $\begin{array}{l}\text { A tail cuff and a pulse sensor (NarcoBiosystems. Houston. TX) were used for recording } \\
\text { the systolic blood pressure. ELISA test for ACE and renin plasma concentration. } \\
\text { Spectrophotometric assay for ACE activity. Contractile response of aortic smooth muscle } \\
\text { was measured in a force transducer (Grass FT.03D. Grass Telefactor. West Warwick. CT. } \\
\text { USA). }\end{array}$ \\
\hline Data format & Raw \\
\hline \multirow[t]{2}{*}{ Parameters for data collection } & $\begin{array}{l}\text { Sixty male SHR rats were used weighed between } 210-290 \mathrm{~g} \text { and had approximately } 10 \\
\text { weeks of age at the beginning of the study. }\end{array}$ \\
\hline & $\begin{array}{l}\text { Rats were housed in stainless steel cages. } 4 \text { animals per cage, with sterilized bedding. } \\
\text { The facility had air conditioning and a } 12 \mathrm{~h} \text { light }-12 \mathrm{~h} \text { dark cycle. Food and water were } \\
\text { provided ad libitum. Tap water was provided in sterilized bottles with stainless steel } \\
\text { nipples. Animals were fed with extruded balanced feed. }\end{array}$ \\
\hline \multirow[t]{2}{*}{ Description of data collection } & $\begin{array}{l}\text { Animals were divided into } 8 \text { groups of } 7-8 \text { animals each, except captopril and aliskirene } \\
\text { groups in which } 4 \text { animals were used. Samples were administered by the orogastric } \\
\text { route with } 2 \mathrm{ml} \text { of each sample dispersed in distilled water. }\end{array}$ \\
\hline & $\begin{array}{l}\text { The systolic blood pressure was measured according to [3]. After surgical procedure the } \\
\text { rat's abdominal aorta was then cannulated to collect a blood sample (roughly } 6 \mathrm{ml} \text { ) in } \\
\text { heparin-coated tubes. The plasma was separated and used for plasma ACE. Renin } \\
\text { concentrations and plasma ACE activity. Also, the thoracic aorta was resected and then } \\
\text { cut into two mm long rings. The ex vivo assay was performed according to [3]. }\end{array}$ \\
\hline \multirow[t]{3}{*}{ Data source location } & Institution: CIDCA. UNLP. CONICET \\
\hline & City/Town/Region: La Plata. Buenos Aires \\
\hline & Country: Argentina \\
\hline Data accessibility & With the article \\
\hline \multirow[t]{4}{*}{ Related research article } & Author's name: Santiago Suárez. Paula Aphalo. Gustavo Rinaldi. María Cristina Añón. \\
\hline & Alejandra Quiroga \\
\hline & $\begin{array}{l}\text { Title: Effect of amaranth proteins on the RAS system. In vitro. in vivo and ex vivo assays } \\
\text { Journal: Food Chemistry }\end{array}$ \\
\hline & DOI: 10.1016/j.foodchem.2019.125601 \\
\hline
\end{tabular}

\footnotetext{
Value of the Data

- This data collects in vitro, in vivo and ex vivo information about amaranth protein and peptides with antihypertensive effect. The interaction of the different approaches is important for understanding the mechanism of action of amaranth peptides.

- The data will help to understand the possible mechanism of action of food peptides on the RAS system.

- Data are useful for researchers and academician to acquire innovative knowledge about the effect of bioactive peptides on RAS system. In addition, data provide new insights and information to consider in the design of novel functional foods with amaranth what could be useful for entrepreneurs and food industry.

- How affect the bioactive peptides on RAS system is a valuable tool to develop a new functional food. Emulsions with amaranth proteins could be a delivery system of antihypertensive peptides with the possibility to enhance the biodisponibility and reach the target organ successfully.
} 


\section{Data description}

Data describes the effect of amaranth protein/peptides on RAS system inquiring into the mechanism of action of these samples using in vitro, in vivo and ex vivo approaches [1].

Treatment groups were:

1. $\mathrm{G}_{\mathrm{W}}$ : Negative control group. Animals treated with water, which did not receive amaranth proteins.

2. GC: Captopril group. Animals treated with captopril, an ACE inhibitor.

3. $G_{A}$ : Aliskiren group. Animals treated with aliskiren, a renin inhibitor.

4. $\mathrm{G}_{\mathrm{API}}$ : API group. Animals treated with amaranth protein isolated (API).

5. $\mathrm{G}_{\mathrm{AH}}$ : AH group. Animals treated with amaranth protein hydrolysate (AH).

6. GVIKP: VIKP group. Animals treated with the synthetic peptide VIKP.

7. $\mathrm{G}_{\mathrm{E}}: \mathrm{w} / \mathrm{o}$ Emulsion group. Animals treated with w/o emulsion.

8. $\mathrm{G}_{\mathrm{E}+\mathrm{VIKP}}$ : w/o Emulsion + VIKP group. Animals treated with w/o emulsion added with VIKP.

In order to compare mean values, a one way analysis of variance (ANOVA) multiple comparisons was applied. The critical significance level was set at $\mathrm{p}<0.05$. All samples were compared to $\mathrm{G}_{\mathrm{W}}$ (negative control group).

Table 1 shows the reduction in SBP values exerted in each experimental group. Data were expressed as the decrease of SBP in $\mathrm{mmHg}$ of animals $3 \mathrm{~h}$ after the administration of each sample with respect to the SBP measured at the beginning of the experiment $\left(\mathrm{SBP}_{3 \mathrm{~h}}-\mathrm{SBP}_{0 \mathrm{hi}}\right) . \Delta \mathrm{P}$ values are presented as mean $\pm \mathrm{SEM}$. Animals belonging to the $\mathrm{G}_{\mathrm{E}}$ and $\mathrm{G}_{\mathrm{E}+\mathrm{VIKP}}$ groups showed the most significant reduction in the SBP reaching reduction values of $42 \pm 2 \mathrm{mmHg}$ and $35 \pm 2 \mathrm{mmHg}$ respectively. The administration of API, AH or VIKP in water as vehicle ( $G_{A P I}, G_{A H}$ y $G_{V I K P}$ groups) caused a reduction in SBP values that was significantly lower than those observed in the groups mentioned above $(25 \pm 14 \mathrm{mmHg}$, $26 \pm 3 \mathrm{mmHg}$ and $21 \pm 3 \mathrm{mmHg}$ respectively.)

Table 2 shows ACE plasma concentration of different groups assayed. This ELISA immunoassay is based on a competitive inhibition. Calibration curve was calculated according to the manufacturer's directions and the values are as follows: $y=0.7089 \mathrm{e}^{(-0.001405 . \mathrm{X})}+0.107$ where " $\mathrm{y}$ " means OD at $450 \mathrm{~nm}$ and " $\mathrm{x}$ " is ACE sample concentration in $\mu \mathrm{g} / \mathrm{ml}$. It can be observed that $\mathrm{G}_{\mathrm{W}}$ group presented extremely low values of ACE levels $(0.17 \pm 0.02 \mu \mathrm{g} / \mathrm{ml})$, whereas ACE concentration in $G_{C} G_{A} G_{E}$ y $G_{E}+$ VIKP groups were 13.6-25.8 times higher than control group. API, AH and VIKP ( $G_{A P I}, G_{A H}, G_{V I K P}$ groups respectively) induced an increase in the ACE levels that was 7.6 to 5.3 times higher than control group $(1.3 \pm 0.2 \mu \mathrm{g} / \mathrm{ml}, 0.90 \pm 0.3 \mu \mathrm{g} / \mathrm{ml}$ and $1.1 \pm 0.3 \mu \mathrm{g} / \mathrm{ml}$ respectively. $\mathrm{p}<0.05)$. The same trend has been observed in studies evaluating synthetic drugs in hypertension treatments [4,5].

Table 3 shows renin plasma concentration in the different samples assayed. Calibration curve obtained was $\mathrm{y}=0.005 \mathrm{x}+0.008$ where " $\mathrm{y}$ " means $\mathrm{OD}$ at $450 \mathrm{~nm}$ and " $\mathrm{x}$ " represented renin concentration in $\mathrm{pg} / \mathrm{ml}$. Only the $\mathrm{G}_{\mathrm{A}}, \mathrm{G}_{\mathrm{API}}$ and $\mathrm{G}_{\mathrm{AH}}$ groups presented differences in plasma renin levels, as compared to $G_{W}$ group. No differences were found in the levels of this enzyme between $G_{C}, G_{E}, G_{V I K P}$ and $G_{E}+$ VIKP groups and the control group $\mathrm{G}_{\mathrm{W}}$.

Table 4 shows \% ACE activity/ $\mu$ g ACE in plasma collected after treatments. Data are expressed as relative to $100 \%$ ACE activity to water control group $\left(G_{W}\right)$. The lowest activity values (4-7\% active ACE/ $\mu \mathrm{g} A C E)$ corresponded to groups $G_{C}, G_{A}, G_{E}$ and $G_{E}+V_{I K P}$, whereas the highest activity values were found in $G_{V I K P}, G_{A H}$ and $G_{A P I}$ groups, which presented $20-13 \%$ active ACE/ $\mu$ g ACE. The administration of different samples decreases the enzymatic activity of ACE, together with an increase in plasma levels (Table 2), probably to counter balance the inhibitory effect exerted by the hypotensive peptides.

Table 5 shows the contractile activity determined in presence of potassium ions. Contractile force was higher in $\mathrm{G}_{W}, \mathrm{G}_{C}$ and $\mathrm{G}_{\mathrm{A}}$ groups (roughly $0.45 \mathrm{~g} / \mathrm{mg}$ ), whereas this activity was significantly lower in the animals belonging to groups $G_{A P I}, G_{V I K P}, G_{E}+$ VIKP, $G_{A H}$ and $G_{E}(0.34-0.29 \mathrm{~g} / \mathrm{mg})$. Upon treating aorta rings with physiological concentrations of norepinephrine, statistically differences were observed in $\mathrm{GVIKP}_{\mathrm{V}}$ and $\mathrm{G}_{\mathrm{E}+\mathrm{VIKP}}$ groups. 
Table 1

Systolic blood pressure before and after treatment. $\Delta \mathrm{P}\left(\mathrm{SBP}_{3 \mathrm{~h}}-\mathrm{SBP}_{0 \mathrm{hi}}\right)$ values are presented as mean $\pm \mathrm{SEM}$.

\begin{tabular}{|c|c|c|c|c|c|c|c|c|c|c|c|c|c|c|c|}
\hline \multicolumn{2}{|c|}{ Treatment group } & \multicolumn{14}{|c|}{$\underline{\mathrm{SBP}(\mathrm{mm} \mathrm{Hg})}$} \\
\hline & & 1 & 2 & 3 & 4 & 5 & 6 & 7 & 8 & 9 & 10 & 11 & 12 & 13 & 14 \\
\hline \multirow[t]{12}{*}{$\mathrm{G}_{\mathrm{W}}$} & & 196 & 281 & 215 & 190 & 188 & 206 & & & & & & & & \\
\hline & & 176 & 280 & 193 & 195 & 177 & 196 & & & & & & & & \\
\hline & & 200 & 273 & 244 & 202 & 170 & 201 & & & & & & & & \\
\hline & average & 191 & 278 & 217 & 196 & 178 & 201 & & & & & & & & \\
\hline & \multicolumn{15}{|c|}{ post intragastric administration } \\
\hline & & 201 & 314 & 208 & 228 & 167 & 188 & & & & & & & & \\
\hline & & 186 & 283 & 179 & 222 & 152 & 187 & & & & & & & & \\
\hline & & 196 & 244 & 156 & 203 & 162 & 250 & & & & & & & & \\
\hline & average & 194 & 280 & 181 & 218 & 160 & 208 & & & & & & & & \\
\hline & $\Delta \mathrm{P}=\mathrm{SBP}_{\mathrm{h}}-\mathrm{SBP}_{0 \mathrm{hi}}$ & 4 & 2 & -36 & 22 & -18 & 7 & & & & & & & & \\
\hline & $\Delta \mathbf{P}$ average & $-3^{a}$ & & & & & & & & & & & & & \\
\hline & SEM & 8 & & & & & & & & & & & & & \\
\hline \multirow[t]{12}{*}{$\mathrm{G}_{\mathrm{A}}$} & & 196 & 185 & 195 & 196 & & & & & & & & & & \\
\hline & & 197 & 183 & 187 & 192 & & & & & & & & & & \\
\hline & & 190 & 186 & 185 & 193 & & & & & & & & & & \\
\hline & average & 194 & 185 & 189 & 194 & & & & & & & & & & \\
\hline & \multicolumn{15}{|c|}{ post intragastric administration } \\
\hline & & 145 & 156 & 139 & 139 & & & & & & & & & & \\
\hline & & 147 & 154 & 140 & 135 & & & & & & & & & & \\
\hline & & 152 & 150 & 145 & 140 & & & & & & & & & & \\
\hline & average & 148 & 153 & 141 & 138 & & & & & & & & & & \\
\hline & $\Delta \mathrm{P}=\mathrm{SBP} 3_{\mathrm{h}}-\mathrm{SBP}_{\mathrm{Oh}}$ & -46 & -31 & -48 & -56 & & & & & & & & & & \\
\hline & $\Delta \mathrm{P}$ average & $-45^{b}$ & & & & & & & & & & & & & \\
\hline & SEM & 5 & & & & & & & & & & & & & \\
\hline \multirow[t]{12}{*}{$G_{C}$} & & 208 & 205 & - & 205 & & & & & & & & & & \\
\hline & & 199 & 205 & - & 193 & & & & & & & & & & \\
\hline & & 199 & 205 & - & 183 & & & & & & & & & & \\
\hline & average & 202 & 205 & & 194 & & & & & & & & & & \\
\hline & \multicolumn{15}{|c|}{ post intragastric administration } \\
\hline & & 166 & 171 & - & 168 & & & & & & & & & & \\
\hline & & 159 & 173 & - & 171 & & & & & & & & & & \\
\hline & & 162 & 177 & - & 163 & & & & & & & & & & \\
\hline & average & 162 & 174 & & 167 & & & & & & & & & & \\
\hline & $\Delta \mathrm{P}=\mathrm{SBP}_{\mathrm{h}}-\mathrm{SBP}_{\mathrm{oh}}$ & -40 & -31 & & -26 & & & & & & & & & & \\
\hline & $\Delta P$ average & $-32^{b}$ & & & & & & & & & & & & & \\
\hline & SEM & 4 & & & & & & & & & & & & & \\
\hline \multirow[t]{12}{*}{$\mathrm{G}_{\mathrm{API}}$} & & 166 & 162 & 197 & 180 & 259 & & & & & & & & & \\
\hline & & 160 & 166 & 208 & 187 & 238 & & & & & & & & & \\
\hline & & 159 & 168 & 204 & 190 & 234 & & & & & & & & & \\
\hline & average & 162 & 165 & 203 & 186 & 244 & & & & & & & & & \\
\hline & \multicolumn{15}{|c|}{ post intragastric administration } \\
\hline & & 153 & 155 & 136 & 140 & 179 & & & & & & & & & \\
\hline & & 145 & 157 & 147 & 154 & 173 & & & & & & & & & \\
\hline & & 158 & 154 & 152 & 162 & 182 & & & & & & & & & \\
\hline & average & 152 & 155 & 145 & 152 & 178 & & & & & & & & & \\
\hline & $\Delta \mathrm{P}=\mathrm{SBP}_{\mathrm{h}}-\mathrm{SBP}_{\mathrm{Oh}}$ & -10 & -10 & -58 & -34 & -66 & & & & & & & & & \\
\hline & $\Delta P$ average & $-35^{b}$ & & & & & & & & & & & & & \\
\hline & SEM & 12 & & & & & & & & & & & & & \\
\hline \multirow[t]{9}{*}{$\mathrm{G}_{\mathrm{AH}}$} & & 181 & 184 & 184 & 162 & 159 & 174 & 203 & 199 & 208 & 183 & 162 & 168 & 170 & 195 \\
\hline & & 184 & 184 & 186 & 159 & 161 & 179 & 192 & 184 & 198 & 173 & 166 & 182 & 181 & 191 \\
\hline & \multirow[t]{2}{*}{ average } & 182 & 177 & 188 & 158 & 164 & 177 & 191 & 173 & 199 & 169 & 160 & 177 & 182 & 185 \\
\hline & & 182 & 182 & 186 & 160 & 161 & 177 & 195 & 185 & 202 & 175 & 163 & 176 & 178 & 190 \\
\hline & post intragastric ad & ministra & tion & & & & & & & & & & & & \\
\hline & & 145 & 166 & 165 & 145 & 140 & 156 & 167 & - & 176 & 146 & 123 & 148 & 150 & 146 \\
\hline & & 156 & 159 & 166 & 156 & 145 & 158 & 161 & - & 177 & 148 & 116 & 141 & 158 & 148 \\
\hline & & 156 & 168 & 158 & 151 & 146 & 155 & 168 & - & 177 & 146 & 129 & 145 & 157 & 139 \\
\hline & average & 152 & 164 & 163 & 151 & 144 & 156 & 165 & - & 177 & 147 & 123 & 145 & 155 & 144 \\
\hline
\end{tabular}


Table 1 (continued)

\begin{tabular}{|c|c|c|c|c|c|c|c|c|c|c|c|c|c|c|c|}
\hline \multicolumn{2}{|c|}{ Treatment group } & \multicolumn{14}{|c|}{$\mathrm{SBP}(\mathrm{mm} \mathrm{Hg})$} \\
\hline & & 1 & 2 & 3 & 4 & 5 & 6 & 7 & 8 & 9 & 10 & 11 & 12 & 13 & 14 \\
\hline & $\begin{array}{l}\Delta \mathrm{P}=\mathrm{SBP}_{\mathrm{h}}-\mathrm{SBP}_{0 \mathrm{~h}} \\
\Delta \mathbf{P} \text { average } \\
\mathbf{S E M}\end{array}$ & $\begin{array}{l}-30 \\
-\mathbf{2 6}^{\mathbf{b}} \\
\mathbf{3}\end{array}$ & -17 & -23 & -9 & -18 & -20 & -30 & - & -25 & -28 & -40 & -31 & -23 & -46 \\
\hline \multirow[t]{12}{*}{$G_{\text {VIKP }}$} & & 209 & 223 & 223 & 221 & 205 & 208 & 201 & 201 & & & & & & \\
\hline & & 206 & 222 & 201 & 218 & 208 & 209 & 199 & 203 & & & & & & \\
\hline & & 205 & 219 & 208 & 219 & 208 & 205 & 203 & 206 & & & & & & \\
\hline & average & 207 & 221 & 211 & 219 & 207 & 207 & 201 & 203 & & & & & & \\
\hline & post intragastric ad & ministra & tion & & & & & & & & & & & & \\
\hline & & 188 & 193 & 183 & 188 & 200 & 193 & 182 & 179 & & & & & & \\
\hline & & 191 & 192 & 181 & 190 & 201 & 183 & 181 & 177 & & & & & & \\
\hline & & 195 & 199 & 177 & 195 & 205 & 188 & 180 & 185 & & & & & & \\
\hline & average & 191 & 195 & 180 & 191 & 202 & 188 & 181 & 180 & & & & & & \\
\hline & $\Delta \mathrm{P}=\mathrm{SBP} 3_{\mathrm{h}}-\mathrm{SBP}_{\mathrm{Oh}}$ & -15 & -27 & -30 & -28 & -5 & -19 & -20 & -23 & & & & & & \\
\hline & $\Delta \mathrm{P}$ average & $-21^{b}$ & & & & & & & & & & & & & \\
\hline & SEM & 3 & & & & & & & & & & & & & \\
\hline \multirow[t]{12}{*}{$\mathrm{G}_{\mathrm{E}}$} & & 188 & 181 & 187 & 183 & 181 & 178 & 190 & 192 & & & & & & \\
\hline & & 186 & 183 & 182 & 187 & 179 & 180 & 196 & 198 & & & & & & \\
\hline & & 191 & 187 & 202 & 185 & 179 & 177 & 191 & 196 & & & & & & \\
\hline & average & 188 & 184 & 190 & 185 & 180 & 178 & 192 & 195 & & & & & & \\
\hline & post intragastric ad & ministra & tion & & & & & & & & & & & & \\
\hline & & 151 & 140 & 142 & 145 & 144 & 142 & 157 & 148 & & & & & & \\
\hline & & 145 & 143 & 142 & 147 & 142 & 141 & 146 & 145 & & & & & & \\
\hline & & 142 & 145 & 149 & 142 & 148 & 145 & 145 & 141 & & & & & & \\
\hline & average & 146 & 143 & 144 & 145 & 145 & 143 & 149 & 145 & & & & & & \\
\hline & $\Delta \mathrm{P}=\mathrm{SBP} 3_{\mathrm{h}}-\mathrm{SBP}_{\mathrm{Oh}}$ & -42 & -41 & -46 & -40 & -35 & -36 & -43 & -51 & & & & & & \\
\hline & $\Delta \mathbf{P}$ average & $-42^{b}$ & & & & & & & & & & & & & \\
\hline & SEM & 2 & & & & & & & & & & & & & \\
\hline \multirow[t]{12}{*}{$\mathrm{G}_{\mathrm{E}+\mathrm{VIKP}}$} & & 173 & 155 & 153 & 151 & 171 & 169 & 171 & & & & & & & \\
\hline & & 175 & 169 & 177 & 174 & 175 & 177 & 175 & & & & & & & \\
\hline & & 169 & 171 & 175 & 173 & 173 & 174 & 173 & & & & & & & \\
\hline & average & 172 & 165 & 168 & 166 & 173 & 173 & 173 & & & & & & & \\
\hline & \multicolumn{15}{|c|}{ post intragastric administration } \\
\hline & & 132 & 138 & 132 & 138 & 132 & 137 & 135 & & & & & & & \\
\hline & & 135 & 136 & 135 & 137 & 134 & 132 & 134 & & & & & & & \\
\hline & & 137 & 134 & 136 & 135 & 137 & 135 & 136 & & & & & & & \\
\hline & average & 135 & 136 & 134 & 137 & 134 & 135 & 135 & & & & & & & \\
\hline & $\Delta \mathrm{P}=\mathrm{SBP} 3_{\mathrm{h}}-\mathrm{SBP}_{\mathrm{Oh}}$ & -38 & -29 & -34 & -29 & -39 & -39 & -38 & & & & & & & \\
\hline & $\Delta \mathbf{P}$ average & $-35^{\mathbf{b}}$ & & & & & & & & & & & & & \\
\hline & SEM & 2 & & & & & & & & & & & & & \\
\hline
\end{tabular}

\section{Experimental design. Materials and methods}

\subsection{Samples}

The following samples were used for in vivo assays:

- Amaranth protein isolate (API) and hydrolysate (AH) prepared from Amaranthus hypochondriacus as described elsewhere [2]. The protein content was $87 \pm 1$ and $57 \pm 2 \% \mathrm{w} / \mathrm{w}$ w.b. for API and AH respectively.

- VIKP peptide, which is a synthetic peptide from 11S amaranth protein. This peptide has inhibitory activity on ACE [6].

- O/W 20:80 emulsions prepared with sunflower oil and 1:1 protein mixture of API and AH at pH 2 with a total protein concentration of $2 \% \mathrm{w} / \mathrm{v}$ with or without VIKP peptide [(API50 + AH50)-2\%+ VIKP and (API50 + AH50)-2\%, respectively]. 
Table 2

Plasma ACE concentration at the end of the $3 \mathrm{~h}$ treatment. Values are presented as mean $\pm \mathrm{SEM}$.

\begin{tabular}{|c|c|c|c|c|c|c|}
\hline Treatment group & Rat & $\mathrm{OD}_{450}(\mathrm{~nm})$ & $\mu \mathrm{g} / \mathrm{ml}$ & Average & SD & SEM \\
\hline \multirow[t]{5}{*}{$\mathrm{G}_{\mathrm{W}}$} & 1 & 0.592 & 0.14 & \multirow[t]{5}{*}{$0.15^{\mathrm{a}}$} & \multirow[t]{5}{*}{0.07} & \multirow[t]{5}{*}{0.03} \\
\hline & 2 & 0.579 & 0.14 & & & \\
\hline & 3 & 0.761 & 0.03 & & & \\
\hline & 4 & 0.464 & 0.24 & & & \\
\hline & 5 & 0.536 & 0.18 & & & \\
\hline \multirow[t]{4}{*}{$\mathrm{G}_{\mathrm{A}}$} & 1 & 0.181 & 0.80 & \multirow[t]{4}{*}{$2.08^{\mathrm{b}}$} & \multirow[t]{4}{*}{0.84} & \multirow[t]{4}{*}{0.42} \\
\hline & 2 & 0.081 & 2.50 & & & \\
\hline & 3 & 0.077 & 2.50 & & & \\
\hline & 4 & 0.076 & 2.50 & & & \\
\hline \multirow[t]{3}{*}{$\mathrm{G}_{\mathrm{C}}$} & 1 & 0.089 & 2.50 & \multirow[t]{3}{*}{$2.50^{\mathrm{b}}$} & \multirow[t]{3}{*}{0} & \multirow[t]{3}{*}{0} \\
\hline & 2 & 0.078 & 2.50 & & & \\
\hline & 3 & 0.09 & 2.50 & & & \\
\hline \multirow[t]{3}{*}{$\mathrm{G}_{\mathrm{API}}$} & 1 & 0.134 & 1.16 & \multirow[t]{3}{*}{$1.35^{\mathrm{b}}$} & \multirow[t]{3}{*}{0.52} & \multirow[t]{3}{*}{0.3} \\
\hline & 2 & 0.11 & 1.94 & & & \\
\hline & 3 & 0.156 & 0.95 & & & \\
\hline \multirow[t]{5}{*}{$\mathrm{G}_{\mathrm{HA}}$} & 1 & 0.184 & 0.79 & \multirow[t]{5}{*}{$1.16^{\mathrm{b}}$} & \multirow[t]{5}{*}{0.88} & \multirow[t]{5}{*}{0.39} \\
\hline & 2 & 0.116 & 1.55 & & & \\
\hline & 3 & 0.095 & 2.50 & & & \\
\hline & 4 & 0.438 & 0.27 & & & \\
\hline & 5 & 0.215 & 0.67 & & & \\
\hline \multirow[t]{4}{*}{$\mathrm{G}_{\mathrm{VIKP}}$} & 1 & 0.151 & 0.99 & \multirow[t]{4}{*}{$0.87^{\mathrm{b}}$} & \multirow[t]{4}{*}{0.64} & \multirow[t]{4}{*}{0.32} \\
\hline & 2 & 0.227 & 0.63 & & & \\
\hline & 3 & 0.539 & 0.18 & & & \\
\hline & 4 & 0.113 & 1.70 & & & \\
\hline \multirow[t]{7}{*}{$\mathrm{G}_{\mathrm{E}}$} & 1 & 0.056 & 2.50 & \multirow[t]{7}{*}{$2.50^{\mathrm{b}}$} & \multirow[t]{7}{*}{0} & 0 \\
\hline & 2 & 0.057 & 2.50 & & & \\
\hline & 3 & 0.057 & 2.50 & & & \\
\hline & 4 & 0.056 & 2.50 & & & \\
\hline & 5 & 0.062 & 2.50 & & & \\
\hline & 6 & 0.057 & 2.50 & & & \\
\hline & 7 & 0.058 & 2.50 & & & \\
\hline $\mathrm{G}_{\mathrm{E}+\mathrm{VIKP}}$ & 1 & 0.062 & 2.50 & $2.29^{\mathrm{b}}$ & 0.46 & 0.2 \\
\hline & 2 & 0.055 & 2.50 & & & \\
\hline & 3 & 0.119 & 1.45 & & & \\
\hline & 4 & 0.06 & 2.50 & & & \\
\hline & 5 & 0.057 & 2.50 & & & \\
\hline
\end{tabular}

Emulsions were prepared according to [2]. Emulsions were frozen at $-80{ }^{\circ} \mathrm{C}$, lyophilized and resuspended as required. Before administration, the resuspended emulsions were homogenized with a magnetic stirring bar.

- Commercial ACE and renin inhibitors (captopril and aliskiren, respectively) were employed as positive controls.

\subsection{In vivo assays}

\subsubsection{Indirect measurement of blood pressure}

The systolic blood pressure was measured according to [3]. In order to determine baseline values, blood pressure values were recorded at least three times on different days for each rat. After recording 
Table 3

Plasma renin concentration at the end of the $3 \mathrm{~h}$ treatment. Values are presented as mean $\pm \mathrm{SEM}$.

\begin{tabular}{|c|c|c|c|c|c|c|c|c|}
\hline \multirow{2}{*}{$\begin{array}{l}\text { Treatment group } \\
G_{W}\end{array}$} & \multirow{2}{*}{$\frac{\text { Rat }}{1}$} & \multicolumn{2}{|c|}{$\mathrm{OD}_{450}(\mathrm{~nm})$} & \multicolumn{2}{|l|}{$\mathrm{pg} / \mathrm{ml}$} & \multirow{2}{*}{$\frac{\text { Average }}{42.4^{\mathrm{a}}}$} & \multirow{2}{*}{$\frac{S D}{9.9}$} & \multirow{2}{*}{$\frac{\text { SEM }}{3.1}$} \\
\hline & & 0.279 & 0.305 & 54.2 & 59.4 & & & \\
\hline & 2 & 0.183 & 0.171 & 35 & 32.6 & & & \\
\hline & 3 & 0.252 & 0.221 & 48.8 & 42.6 & & & \\
\hline & 4 & 0.223 & 0.239 & 43 & 46.2 & & & \\
\hline & 5 & 0.158 & 0.171 & 30 & 32.6 & & & \\
\hline \multirow[t]{3}{*}{$\mathrm{G}_{\mathrm{A}}$} & 1 & 0.199 & 0.175 & 38.2 & 33.4 & $34.2^{\mathrm{b}}$ & 4.7 & 1.9 \\
\hline & 2 & 0.216 & 0.16 & 41.6 & 30.4 & & & \\
\hline & 3 & 0.166 & 0.158 & 31.6 & 30 & & & \\
\hline \multirow[t]{3}{*}{$\mathrm{G}_{\mathrm{C}}$} & 1 & 0.193 & 0.178 & 37 & 34 & $35.1^{\mathrm{a}}$ & 3.4 & 1.4 \\
\hline & 2 & 0.176 & 0.159 & 33.6 & 30.2 & & & \\
\hline & 3 & 0.21 & 0.185 & 40.4 & 35.4 & & & \\
\hline \multirow[t]{4}{*}{$\mathrm{G}_{\mathrm{API}}$} & 1 & 0.13 & 0.151 & 24.4 & 28.6 & $34.0^{\mathrm{b}}$ & 7.3 & 2.5 \\
\hline & 2 & 0.228 & 0.222 & 44 & 42.8 & & & \\
\hline & 3 & 0.137 & 0.177 & 25.8 & 33.8 & & & \\
\hline & 4 & 0.193 & 0.184 & 37 & 35.2 & & & \\
\hline \multirow[t]{4}{*}{$\mathrm{G}_{\mathrm{AH}}$} & 1 & 0.157 & 0.155 & 29.8 & 29.4 & $30.7^{\mathrm{b}}$ & 4.1 & 1.9 \\
\hline & 2 & 0.193 & 0.152 & 37 & 28.8 & & & \\
\hline & 3 & 0.141 & 0.143 & 26.6 & 27 & & & \\
\hline & 4 & 0.194 & 0.157 & 37.2 & 29.8 & & & \\
\hline \multirow[t]{3}{*}{$G_{\mathrm{VIKP}}$} & 1 & 0.192 & 0.15 & 36.8 & 28.4 & $34.9^{\mathrm{a}}$ & 4.7 & 1.9 \\
\hline & 2 & 0.221 & 0.17 & 42.6 & 32.4 & & & \\
\hline & 3 & 0.18 & 0.181 & 34.4 & 34.6 & & & \\
\hline \multirow[t]{3}{*}{$\mathrm{G}_{\mathrm{E}}$} & 1 & 0.207 & 0.167 & 39.8 & 31.8 & $35.1^{\mathrm{a}}$ & 4.7 & 1.9 \\
\hline & 2 & 0.163 & 0.159 & 31 & 30.2 & & & \\
\hline & 3 & 0.212 & 0.192 & 40.8 & 36.8 & & & \\
\hline \multirow[t]{4}{*}{$\mathrm{G}_{\mathrm{E}+\mathrm{VIKP}}$} & 1 & 0.237 & 0.209 & 45.8 & 40.2 & $36.3^{\mathrm{a}}$ & 9.6 & 3 \\
\hline & 2 & 0.192 & 0.175 & 36.8 & 33.4 & & & \\
\hline & 3 & 0.236 & 0.227 & 45.6 & 43.8 & & & \\
\hline & 4 & 0.124 & 0.115 & 23.2 & 21.4 & & & \\
\hline
\end{tabular}

the last baseline blood pressure value, an aqueous suspension of each sample was administered to each animal. Three hours after the administration, blood pressure values were recorded with a tail cuff and a pulse sensor (NarcoBiosystems, Houston, TX).

\subsubsection{Determination of plasma ACE and renin concentrations}

A commercial ELISA kit (Rat Angiotensin converting enzyme MBS703086, MyBioSource, CA, USA) was employed to determine ACE concentration according to manufacturer's directions. This immunoassay is based on a competitive inhibition. Briefly, microtitre plates are coated with ACE. Samples and standards are incubated together with an anti-ACE HRP-labeled conjugate to generate the competition. Plasma renin concentration was determined with a commercial ELISA kit (Rat renin ELISA kit MBS041519 MyBioSource) following the manufacturer's directions. This immunoassay is a direct ELISA, which has an analytical measurement range of $6.25-200 \mathrm{pg} / \mathrm{ml}$. The final colour reaction was read in a microtiter plate reader (Biotek Synergy HT, Winooski, VT, USA) at $450 \mathrm{~nm}$.

\subsubsection{Determination of plasma ACE activities}

ACE-inhibitory activity was assayed according to [2]. Briefly, to determine the enzymatic activity, 50 $\mu \mathrm{l}$ of buffer [0.2M sodium borate $\mathrm{pH} 8.3 ; 2 \mathrm{M} \mathrm{NaCl}$ ), $25 \mu \mathrm{l}$ of milli Q water, $25 \mu \mathrm{l}$ of the commercial enzyme (maximum activity control)], or plasma samples were incubated with $100 \mu$ of synthetic substrate (HHL) at $37^{\circ} \mathrm{C}$ for $30 \mathrm{~min}$. The reaction was stopped by heating the mixture over a water bath at $90{ }^{\circ} \mathrm{C}$ for $15 \mathrm{~min}$. After cooling, $600 \mu \mathrm{l}$ of $0.2 \mathrm{M}$ potassium pH 8.2 and $515 \mu \mathrm{l}$ of colour reagent, which reacts with the hippuric acid generated during the enzymatic reaction, were added and stirred 
Table 4

Plasma ACE activity. These data are expressed as relative to $100 \%$ ACE activity to water control group (Cw). Data are presented as mean \pm SEM.

\begin{tabular}{|c|c|c|c|c|c|c|c|c|c|c|c|}
\hline \multirow{2}{*}{$\begin{array}{l}\text { Treatment group } \\
G_{W}\end{array}$} & \multirow{2}{*}{$\frac{\text { Rats }}{1}$} & \multicolumn{2}{|c|}{$\mathrm{DO}_{382}(\mathrm{~nm})$} & \multirow{2}{*}{$\begin{array}{l}\mu \mathrm{g} \text { enzyme } \\
0.0037\end{array}$} & \multicolumn{2}{|c|}{$\mathrm{DO}_{382} / \mu \mathrm{g}$ enzyme } & \multicolumn{2}{|c|}{ Activity (\%)/ $\mu \mathrm{g}$ enzyme } & \multirow{2}{*}{$\frac{\text { Average }}{100.0^{\mathrm{a}}}$} & \multirow{2}{*}{$\frac{S D}{4.8}$} & \multirow{2}{*}{$\frac{\text { SEM }}{0.01}$} \\
\hline & & 0.794 & 0.773 & & 214.6 & 208.8 & 104.5 & 101.7 & & & \\
\hline & 2 & 0.790 & 0.725 & 0.0037 & 213.4 & 196.0 & 103.9 & 95.5 & & & \\
\hline & 3 & 0.689 & 0.752 & 0.0037 & 186.3 & 203.3 & 90.7 & 99.0 & & & \\
\hline & 4 & 0.785 & 0.798 & 0.0037 & 212.2 & 215.8 & 103.4 & 105.1 & & & \\
\hline & 5 & 0.768 & 0.721 & 0.0037 & 207.7 & 195.0 & 101.2 & 95.0 & & & \\
\hline \multirow[t]{4}{*}{$\mathrm{G}_{\mathrm{A}}$} & 1 & 0.845 & 0.771 & 0.0519 & 16.3 & 14.9 & 7.8 & 7.1 & $7.3^{\mathrm{b}}$ & 0.2 & 0.01 \\
\hline & 2 & 0.786 & 0.781 & 0.0519 & 15.1 & 15.0 & 7.3 & 7.2 & & & \\
\hline & 3 & 0.791 & 0.781 & 0.0519 & 15.2 & 15.0 & 7.3 & 7.2 & & & \\
\hline & 4 & 0.771 & 0.801 & 0.0519 & 14.9 & 15.4 & 7.1 & 7.4 & & & \\
\hline \multirow[t]{3}{*}{$\mathrm{G}_{\mathrm{C}}$} & 1 & 0.733 & 0.790 & 0.0625 & 11.7 & 12.6 & 5.6 & 6.1 & $5.8^{\mathrm{b}}$ & 0.2 & 0.01 \\
\hline & 2 & 0.764 & 0.785 & 0.0625 & 12.2 & 12.6 & 5.9 & 6.0 & & & \\
\hline & 3 & 0.710 & 0.756 & 0.0625 & 11.4 & 12.1 & 5.5 & 5.8 & & & \\
\hline \multirow[t]{4}{*}{$\mathrm{G}_{\mathrm{API}}$} & 1 & 0.723 & - & 0.0338 & 21.4 & - & 10.3 & & $10.7^{\mathrm{b}}$ & 0.3 & 0.01 \\
\hline & 2 & 0.761 & - & 0.0338 & 22.5 & - & 10.8 & & & & \\
\hline & 3 & 0.757 & 0.785 & 0.0338 & 22.4 & 23.2 & 10.8 & 11.2 & & & \\
\hline & 4 & 0.748 & 0.739 & 0.0338 & 22.1 & 21.9 & 10.6 & 10.5 & & & \\
\hline \multirow[t]{5}{*}{$\mathrm{G}_{\mathrm{AH}}$} & 1 & 0.747 & 0.756 & 0.0218 & 34.3 & 34.7 & 16.5 & 16.7 & $16.9^{\mathrm{b}}$ & 0.5 & 0.01 \\
\hline & 2 & 0.716 & 0.752 & 0.0218 & 32.8 & 34.5 & 15.8 & 16.6 & & & \\
\hline & 3 & 0.791 & 0.747 & 0.0218 & 36.3 & 34.3 & 17.5 & 16.5 & & & \\
\hline & 4 & 0.801 & 0.776 & 0.0218 & 36.7 & 35.6 & 17.7 & 17.2 & & & \\
\hline & 5 & 0.776 & 0.771 & 0.0218 & 35.6 & 35.4 & 17.2 & 17.0 & & & \\
\hline \multirow[t]{7}{*}{$G_{V I K P}$} & 1 & 0.794 & 0.799 & 0.0218 & 36.4 & 36.7 & 17.5 & 17.6 & $17.7^{\mathrm{b}}$ & 0.5 & 0.01 \\
\hline & 2 & 0.809 & 0.799 & 0.0218 & 37.1 & 36.7 & 17.8 & 17.6 & & & \\
\hline & 3 & 0.744 & 0.819 & 0.0218 & 34.1 & 37.6 & 16.4 & 18.1 & & & \\
\hline & 4 & 0.804 & 0.799 & 0.0218 & 36.9 & 36.7 & 17.7 & 17.6 & & & \\
\hline & 5 & 0.840 & 0.814 & 0.0218 & 38.6 & 37.3 & 18.5 & 17.9 & & & \\
\hline & 6 & 0.824 & 0.809 & 0.0218 & 37.8 & 37.1 & 18.2 & 17.8 & & & \\
\hline & 7 & 0.799 & 0.785 & 0.0218 & 36.7 & 36.0 & 17.6 & 17.3 & & & \\
\hline \multirow[t]{7}{*}{$\mathrm{G}_{\mathrm{E}}$} & 1 & 0.742 & 0.765 & 0.0625 & 11.9 & 12.2 & 5.7 & 5.9 & $5.8^{\mathrm{b}}$ & 0.1 & 0 \\
\hline & 2 & 0.785 & 0.790 & 0.0625 & 12.6 & 12.6 & 6.0 & 6.1 & & & \\
\hline & 3 & 0.742 & 0.756 & 0.0625 & 11.9 & 12.1 & 5.7 & 5.8 & & & \\
\hline & 4 & 0.751 & 0.756 & 0.0625 & 12.0 & 12.1 & 5.8 & 5.8 & & & \\
\hline & 5 & 0.765 & 0.770 & 0.0625 & 12.2 & 12.3 & 5.9 & 5.9 & & & \\
\hline & 6 & 0.742 & 0.747 & 0.0625 & 11.9 & 12.0 & 5.7 & 5.8 & & & \\
\hline & 7 & 0.725 & 0.775 & 0.0625 & 11.6 & 12.4 & 5.6 & 6.0 & & & \\
\hline \multirow[t]{5}{*}{$\mathrm{G}_{\mathrm{E}+\mathrm{VIKP}}$} & 1 & 0.785 & 0.684 & 0.0573 & 13.7 & 11.9 & 6.6 & 5.7 & $6.4^{\mathrm{b}}$ & 0.3 & 0.01 \\
\hline & 2 & 0.770 & 0.785 & 0.0573 & 13.4 & 13.7 & 6.5 & 6.6 & & & \\
\hline & 3 & 0.775 & 0.761 & 0.0573 & 13.5 & 13.3 & 6.5 & 6.4 & & & \\
\hline & 4 & 0.756 & 0.765 & 0.0573 & 13.2 & 13.4 & 6.4 & 6.4 & & & \\
\hline & 5 & 0.821 & 0.765 & 0.0573 & 14.3 & 13.4 & 6.9 & 6.4 & & & \\
\hline
\end{tabular}

vigorously with a vortex and then centrifuged for $10 \mathrm{~min}$ at $20^{\circ} \mathrm{C}$ and $3000 \times \mathrm{g}$. The absorbance was measured at $382 \mathrm{~nm}$ in a spectrophotometer (Beckman DU 650). The reaction blank was obtained by incubating the synthetic substrate with neither the plasma samples nor the enzyme, completing the reaction volume with milli Q water. Reaction blanks without the substrate (HHL was replaced by $100 \mu 1$ of borate buffer) and containing plasma samples were also included. Controls containing plasma samples and captopril were also assayed.

\subsubsection{Ex vivo experiments}

During the surgical procedure employed to obtain blood samples, the thoracic aorta was resected and placed in saline solution bubbled with $5 \% \mathrm{CO}_{2}$ and $95 \% \mathrm{O}_{2}$. The adjacent connective tissue was carefully removed avoiding distention of the vessel and damage to the endothelium. The aorta was 
Table 5

Effect of different samples on isolated aortic rings contracted by exposure to a high concentration of: Potassium ion ( $80 \mathrm{mM})$ and Norepinephrine $\left(10^{-6} \mathrm{M}\right)$. $\mathrm{F}_{\mathrm{b} 1}(\mathrm{~g})$ : basal force before $\mathrm{K}$ addition. $\mathrm{F}_{\mathrm{b} 2}(\mathrm{~g})$ : basal force before $\mathrm{N}$ addition. $\mathrm{F}_{\mathrm{c}}(\mathrm{g})$ : contractile force. $\mathrm{N}$ : norepinephrine. K: potassium ion. AW: aorta weight. Data are presented as mean \pm SEM.

\begin{tabular}{|c|c|c|c|c|c|c|c|c|}
\hline Treatment group & Rats & $\mathrm{F}_{\mathrm{b} 1}(\mathrm{~g})$ & $\mathrm{F}_{\mathrm{c}} \mathrm{K}(\mathrm{g})$ & $\mathrm{F}_{\mathrm{b} 2}(\mathrm{~g})$ & $\mathrm{F}_{\mathrm{c}} \mathrm{N}(\mathrm{g})$ & $\mathrm{AW}(\mathrm{mg})$ & $\mathrm{F}_{\mathrm{c}} \mathrm{K}(\mathrm{g} / \mathrm{mg})$ & $\mathrm{F}_{\mathrm{c}} \mathrm{N}(\mathrm{g} / \mathrm{mg})$ \\
\hline $\mathrm{G}_{\mathrm{w}}$ & $\begin{array}{l}1 \\
2 \\
3 \\
4 \\
5 \\
6 \\
\text { average } \\
\text { SD } \\
\text { SEM }\end{array}$ & $\begin{array}{l}0 \\
0.07 \\
-0.08 \\
-0.13 \\
-0.03 \\
0.02\end{array}$ & $\begin{array}{l}1.15 \\
1.28 \\
0.8 \\
1.08 \\
1.18 \\
1.2\end{array}$ & $\begin{array}{l}0.18 \\
0.22 \\
0.14 \\
0.12 \\
0.02 \\
0.14\end{array}$ & $\begin{array}{l}2.06 \\
1.79 \\
1.2 \\
1.25 \\
1.09 \\
1.36\end{array}$ & $\begin{array}{l}2.69 \\
2.33 \\
2.24 \\
3.4 \\
3.21 \\
2.58\end{array}$ & $\begin{array}{l}0.428 \\
0.519 \\
0.393 \\
0.356 \\
0.377 \\
0.457 \\
\mathbf{0 . 4 2} \\
\mathbf{0 . 0 6} \\
\mathbf{0 . 0 2}\end{array}$ & $\begin{array}{l}0.699 \\
0.674 \\
0.473 \\
0.332 \\
0.333 \\
0.473 \\
\mathbf{0 . 5 0}^{\mathbf{a}} \\
\mathbf{0 . 1 7} \\
\mathbf{0 . 0 7}\end{array}$ \\
\hline $\mathrm{G}_{\mathrm{A}}$ & $\begin{array}{l}1 \\
2 \\
3 \\
4 \\
\text { average } \\
\text { SD } \\
\text { SEM }\end{array}$ & $\begin{array}{l}-0.042 \\
-0.07 \\
0.021 \\
0.021\end{array}$ & $\begin{array}{l}1.113 \\
1.015 \\
1.113 \\
0.742\end{array}$ & $\begin{array}{l}0.056 \\
-0.007 \\
0.0035 \\
0.049\end{array}$ & $\begin{array}{l}1.253 \\
1.239 \\
1.253 \\
1.001\end{array}$ & $\begin{array}{l}3.47 \\
2.00 \\
2.42 \\
1.95\end{array}$ & $\begin{array}{l}0.333 \\
0.543 \\
0.451 \\
0.370 \\
\mathbf{0 . 4 4} \\
\mathbf{0 . 1 1} \\
\mathbf{0 . 0 5}\end{array}$ & $\begin{array}{l}0.345 \\
0.623 \\
0.516 \\
0.538 \\
\mathbf{0 . 4 9}^{\mathbf{a}} \\
\mathbf{0 . 1 4} \\
\mathbf{0 . 0 7}\end{array}$ \\
\hline $\mathrm{G}_{\mathrm{C}}$ & $\begin{array}{l}1 \\
2 \\
3 \\
\text { average } \\
\text { SD } \\
\text { SEM }\end{array}$ & $\begin{array}{l}0.03 \\
0.07 \\
-0.05\end{array}$ & $\begin{array}{l}1.68 \\
1.54 \\
1.34\end{array}$ & $\begin{array}{l}-0.15 \\
0.59 \\
0\end{array}$ & $\begin{array}{l}1.29 \\
1.85 \\
1.57\end{array}$ & $\begin{array}{l}3.95 \\
3.45 \\
3.12\end{array}$ & $\begin{array}{l}0.418 \\
0.426 \\
0.446 \\
\mathbf{0 . 4 3} \\
\mathbf{0 . 0 1} \\
\mathbf{0 . 0 1}\end{array}$ & $\begin{array}{l}0.365 \\
0.365 \\
0.503 \\
\mathbf{0 . 4 1}^{\mathbf{a}} \\
\mathbf{0 . 0 8} \\
\mathbf{0 . 0 5}\end{array}$ \\
\hline $\mathrm{G}_{\mathrm{API}}$ & $\begin{array}{l}1 \\
2 \\
3 \\
4 \\
5 \\
6 \\
\text { average } \\
\text { SD } \\
\text { SEM }\end{array}$ & $\begin{array}{l}2.1 \\
1.98 \\
2.08 \\
0.1 \\
0 \\
0\end{array}$ & $\begin{array}{l}2.95 \\
2.67 \\
3.29 \\
0.9 \\
0.9 \\
1\end{array}$ & $\begin{array}{l}2.1 \\
2.19 \\
2.29 \\
0.3 \\
0.1 \\
0.2\end{array}$ & $\begin{array}{l}3.48 \\
3.21 \\
3.73 \\
1.2 \\
1.1 \\
1.3\end{array}$ & $\begin{array}{l}2.56 \\
3.12 \\
3.94 \\
2.85 \\
1.9 \\
2.63\end{array}$ & $\begin{array}{l}0.332 \\
0.221 \\
0.307 \\
0.281 \\
0.474 \\
0.380 \\
\mathbf{0 . 3 3 2} \mathbf{b}^{\mathbf{b}} \\
\mathbf{0 . 0 8 7} \\
\mathbf{0 . 0 3 6}\end{array}$ & $\begin{array}{l}0.539 \\
0.327 \\
0.365 \\
0.316 \\
0.526 \\
0.418 \\
\mathbf{0 . 4 1 5}^{\mathbf{a}} \\
\mathbf{0 . 0 9 8} \\
\mathbf{0 . 0 4 0}\end{array}$ \\
\hline $\mathrm{G}_{\mathrm{AH}}$ & $\begin{array}{l}1 \\
2 \\
3 \\
4 \\
5 \\
6 \\
7 \\
8 \\
9 \\
10 \\
11 \\
12 \\
13 \\
\text { average } \\
\text { SD } \\
\text { SEM }\end{array}$ & $\begin{array}{l}-0.1 \\
-0.1 \\
0 \\
-0.02 \\
0.06 \\
-0.04 \\
0.07 \\
0.03 \\
-0.04 \\
-0.01 \\
0.04 \\
-0.07 \\
0.02\end{array}$ & $\begin{array}{l}1.2 \\
1.5 \\
0.9 \\
0.5995 \\
0.6655 \\
0.8525 \\
0.528 \\
0.7645 \\
0.72 \\
0.8262 \\
0.624 \\
0.402 \\
0.520\end{array}$ & $\begin{array}{l}0.4 \\
0.3 \\
0.2 \\
-0.275 \\
-0.06 \\
-0.099 \\
-0.165 \\
-0.0495 \\
-0.126 \\
0.0108 \\
0.042 \\
-0.042 \\
0.016\end{array}$ & $\begin{array}{l}1.5 \\
1.8 \\
1.1 \\
0.8085 \\
0.9075 \\
1.089 \\
0.759 \\
0.935 \\
0.894 \\
10.476 \\
0.72 \\
0.528 \\
0.668\end{array}$ & $\begin{array}{l}2.50 \\
2.98 \\
2.38 \\
2.06 \\
1.90 \\
1.91 \\
2.24 \\
2.20 \\
2.22 \\
2.41 \\
2.01 \\
1.87 \\
3.28\end{array}$ & $\begin{array}{l}0.52 \\
0.537 \\
0.378 \\
0.301 \\
0.319 \\
0.467 \\
0.204 \\
0.334 \\
0.342 \\
0.347 \\
0.291 \\
0.252 \\
0.152 \\
\mathbf{0 . 3 4 0} \\
\mathbf{0 . 1 1} \\
\mathbf{0 . 0 3}\end{array}$ & $\begin{array}{l}0.44 \\
0.503 \\
0.378 \\
0.526 \\
0.509 \\
0.622 \\
0.413 \\
0.448 \\
0.459 \\
0.430 \\
0.337 \\
0.305 \\
0.199 \\
\mathbf{0 . 4 3} \\
\mathbf{0 . 1 1}^{\mathbf{a}} \\
\mathbf{0 . 0 3}\end{array}$ \\
\hline$G_{V I K P}$ & $\begin{array}{l}1 \\
2 \\
3 \\
4 \\
5 \\
6 \\
7\end{array}$ & $\begin{array}{l}0.014 \\
0.077 \\
-0.098 \\
0.014 \\
0.02 \\
0 \\
0\end{array}$ & $\begin{array}{l}0.868 \\
0.861 \\
0.84 \\
1.162 \\
1.27 \\
1.056 \\
1.24\end{array}$ & $\begin{array}{l}0.049 \\
0.077 \\
-0.049 \\
0.07 \\
-0.15 \\
0 \\
-0.016\end{array}$ & $\begin{array}{l}1.316 \\
1.239 \\
1.204 \\
1.61 \\
0.84 \\
1.04 \\
1.1\end{array}$ & $\begin{array}{l}3.15 \\
3.00 \\
3.30 \\
2.86 \\
2.75 \\
2.81 \\
3.37\end{array}$ & $\begin{array}{l}0.271 \\
0.261 \\
0.284 \\
0.401 \\
0.455 \\
0.376 \\
0.368\end{array}$ & $\begin{array}{l}0.402 \\
0.387 \\
0.380 \\
0.538 \\
0.360 \\
0.370 \\
0.331\end{array}$ \\
\hline
\end{tabular}

(continued on next page) 
Table 5 (continued)

\begin{tabular}{|c|c|c|c|c|c|c|c|c|}
\hline Treatment group & Rats & $\mathrm{F}_{\mathrm{b} 1}(\mathrm{~g})$ & $\mathrm{F}_{\mathrm{c}} \mathrm{K}(\mathrm{g})$ & $\mathrm{F}_{\mathrm{b} 2}(\mathrm{~g})$ & $F_{c} N(g)$ & AW (mg) & $\mathrm{F}_{\mathrm{c}} \mathrm{K}(\mathrm{g} / \mathrm{mg})$ & $\mathrm{F}_{\mathrm{c}} \mathrm{N}(\mathrm{g} / \mathrm{mg})$ \\
\hline & $\begin{array}{l}8 \\
\text { average } \\
\text { SD } \\
\text { SEM }\end{array}$ & 0.056 & 1.008 & -0.04 & 0.9 & 3.61 & $\begin{array}{l}0.264 \\
0.335^{\mathbf{b}} \\
0.069 \\
\mathbf{0 . 0 3}\end{array}$ & $\begin{array}{l}0.260 \\
0.379^{b} \\
0.073 \\
0.03\end{array}$ \\
\hline $\mathrm{G}_{\mathrm{E}}$ & $\begin{array}{l}1 \\
2 \\
3 \\
4 \\
5 \\
6 \\
7 \\
\text { average } \\
\text { SD } \\
\text { SEM }\end{array}$ & $\begin{array}{l}0.04 \\
-0.02 \\
0.0495 \\
0.0162 \\
0.1 \\
0.24 \\
0.26\end{array}$ & $\begin{array}{l}0.979 \\
0.715 \\
0.891 \\
0.6696 \\
0.476 \\
0.567 \\
0.616\end{array}$ & $\begin{array}{l}-0.0825 \\
-0.044 \\
0 \\
-0.1134 \\
-0.413 \\
-0.287 \\
0.056\end{array}$ & $\begin{array}{l}1.094 \\
0.902 \\
1.144 \\
0.8748 \\
0.826 \\
0.623 \\
1.008\end{array}$ & $\begin{array}{l}2.42 \\
1.61 \\
2.02 \\
2.30 \\
2.85 \\
2.00 \\
2.09\end{array}$ & $\begin{array}{l}0.388 \\
0.457 \\
0.417 \\
0.284 \\
0.132 \\
0.164 \\
0.170 \\
\mathbf{0 . 2 8 7 ^ { b }} \\
\mathbf{0 . 1 3 5} \\
\mathbf{0 . 0 5}\end{array}$ & $\begin{array}{l}0.486 \\
0.588 \\
0.566 \\
0.430 \\
0.435 \\
0.455 \\
0.456 \\
\mathbf{0 . 4 8 8} \\
\mathbf{0 . 0 6 4} \\
\mathbf{0 . 0 2}\end{array}$ \\
\hline $\mathrm{G}_{\mathrm{E}+\mathrm{VIKP}}$ & $\begin{array}{l}1 \\
2 \\
3 \\
4 \\
5 \\
6 \\
7 \\
\text { average } \\
\text { SD } \\
\text { SEM }\end{array}$ & $\begin{array}{l}-0.012 \\
0.0616 \\
-0.03 \\
0.028 \\
0.02 \\
-0.01 \\
-0.08\end{array}$ & $\begin{array}{l}0.69 \\
0.6496 \\
0.78 \\
0.8008 \\
1.2 \\
1.05 \\
1.1\end{array}$ & $\begin{array}{l}-0.06 \\
-0.028 \\
-0.115 \\
-0.056 \\
-0.11 \\
-0.11 \\
-0.17\end{array}$ & $\begin{array}{l}0.768 \\
0.6216 \\
0.855 \\
0.9968 \\
0.75 \\
0.642 \\
0.602\end{array}$ & $\begin{array}{l}2.73 \\
2.44 \\
2.79 \\
2.60 \\
2.70 \\
3.13 \\
3.01\end{array}$ & $\begin{array}{l}0.257 \\
0.241 \\
0.290 \\
0.297 \\
0.437 \\
0.339 \\
0.392 \\
\mathbf{0 . 3 2 2} \\
\mathbf{0 . 0 7 2} \\
\mathbf{0 . 0 3}\end{array}$ & $\begin{array}{l}0.303 \\
0.266 \\
0.348 \\
0.405 \\
0.319 \\
0.240 \\
0.256 \\
\mathbf{0 . 3 0 5} \\
\mathbf{0 . 0 5 8} \\
\mathbf{0 . 0 2}\end{array}$ \\
\hline
\end{tabular}

then cut into $2 \mathrm{~mm}$ long rings. Assay was performed according to [3]. The rings were gently suspended between two stainless steel wires in a water-jacketed organ baths kept at $37^{\circ} \mathrm{C}$ and filled with saline solution, bubbled with a mixture of $5 \% \mathrm{CO}_{2}$ and $95 \% \mathrm{O}_{2}$, giving a $\mathrm{pH}$ of 7.40 . The lower wire was fixed to a vertical plastic rod immersed in the organ bath, while the upper one was rigidly connected to a force transducer (Grass FT.03D, Grass Telefactor, West Warwick, CT, USA). Preparations were then stretched to obtain a passive force of $2 \mathrm{~g}$ and stabilized during $1 \mathrm{~h}$, changing the solution in the chamber every 20 min. Tissue rings were then exposed to a solution containing $80 \mathrm{mM}$ potassium or norepinephrine $10^{-6}$ $\mathrm{M}$. For each condition, the contractile response was recorded. At the end of the experiment, tissue rings were dried on filter paper and weighed on a precision scale. The contraction intensity was calculated as the quotient between strength and the weight of the ring $(\mathrm{mgF} / \mathrm{mg})$.

\section{Acknowledgments}

This work was supported by Agencia Nacional de Promoción Científica y Tecnológica (ANPCyT, Argentina), Project PICT-2012-0937.

\section{Conflict of Interest}

The authors declare that they have no known competing financial interests or personal relationships that could have appeared to influence the work reported in this paper.

\section{Appendix A. Supplementary data}

Supplementary data to this article can be found online at https://doi.org/10.1016/j.dib.2020.105168.

\section{References}

[1] S. Suárez, P. Aphalo, G. Rinaldi, M.C. Añón, A. Quiroga, Effect of amaranth proteins on the RAS system. In vitro, in vivo and ex vivo assays, Food Chem. 308 (2020) 125601. 
[2] S. Suárez, M.C. Añón, Amaranth proteins emulsions as delivery system of Angiotensin-I converting enzyme inhibitory peptides, Food Hydrocolloids 90 (2019) 154-161.

[3] M. Fritz, B. Vecchi, G. Rinaldi, M.C. Añón, Amaranth seed protein hydrolysates have in vivo and in vitro antihypertensive activity, Food Chem. 126 (2011) 878-884.

[4] S.Y. Chai, R. Perich, B. Jackson, F.A.O. Mendelsohn, C.I. Johnston, Acute and chronic effects of angiotensin-converting enzyme inhibitors on tissue angiotensin-converting enzyme, Clin. Exp. Pharmacol. Physiol. 19 (1992) 7-12.

[5] M. Kohzuki, C.I. Johnston, S.Y. Chai, B. Jackson, R. Perich, D. Paxton, F.A. Mendelsohn, Measurement of angiotensin converting enzyme induction and inhibition using quantitative in vitro autoradiography tissue selective induction after chronic lisinopril treatment, J. Hypertens. 9 (1991) 579-587.

[6] B. Vecchi, M.C. Añón, ACE inhibitory tetrapeptides from Amaranthus hypochondriacus 11S globulin, Phytochemistry 70 (2009) 864-870. 